\title{
T1 mapping using a saturation recovery single- shot acquisition at 3 Tesla MRI in differentiation of normal myocardium from hypertrophic cardiomyopathy
}

\author{
Ryo Ogawa ${ }^{* *}$, Tomoyuki Kido ${ }^{1}$, Masashi Nakamura², Teruhito Kido ${ }^{1}$, Akiyoshi Ogimoto ${ }^{1}$, Masao Miyagawa', \\ Teruhito Mochizuki ${ }^{1}$
}

From 19th Annual SCMR Scientific Sessions

Los Angeles, CA, USA. 27-30 January 2016

\section{Background}

Background Diffuse myocardial fibrosis is characteristic feature of hypertrophic cardiomyopathy (HCM). T1 mapping may enable non-invasive evaluation of diffuse myocardial fibrosis in HCM. There have been many studies that T1 mapping using a modified Look-Locker inversion recovery (MOLLI) sequence. However, there have been few studies using a SASHA for T1 mapping at 3 Tesla MRI. The purpose of this study was to examine $\mathrm{T} 1$ values using a SASHA, in the differentiation between healthy controls and HCM patients.

\section{Methods}

The clinical diagnosis of HCM was established via echocardiography, Cardiac Magnetic Resonance, electrocardiogram, laboratory examination, family history and other clinical data. Twenty patients with HCM and ten healthy controls, underwent left-ventricular T1 mapping in 3 short-axis slices (basal, mid, apex) at 3 Tesla MRI
(Philips Achieva). For T1 mapping, SASHA was used before and at 10 minutes after injection of $0.1 \mathrm{mmol} / \mathrm{kg}$ of gadolinium contrast. T1 values were quantified for 4 segments (anterior, lateral, septum, inferior) of 3 shortaxis slices.

\section{Results}

Native T1 values were significantly longer in HCM compared with healthy controls (HCM $1377 \pm 72 \mathrm{~ms}$ vs healthy controls $1285 \pm 49 \mathrm{~ms} ; \mathrm{p}<0.01$ ). Post-contrast T1 values were significantly shorter in HCM compared with healthy controls (HCM $840 \pm 64$ ms vs healthy controls $908 \pm 94 \mathrm{~ms} ; \mathrm{p}<0.01)$. A cutoff value of 1363 $\mathrm{ms}$ for native $\mathrm{T} 1$ values allowed differentiation between healthy and abnormal segments (HCM) with a sensitivity of $57 \%$, specificity of $92 \%$, accuracy of $69 \%$, and an area under the curve (AUC) of 0.77. On the other hand, post-contrast $\mathrm{T} 1$ values showed lower discriminative ability than native $\mathrm{T} 1$ values.

\section{Table 1 T1 values in HCM and healthy controls}

\begin{tabular}{ccc}
\hline & native T1 values (ms) (median \pm quartile deviation) & post T1 values (ms) (median \pm quartile deviation) \\
\hline HCM (240 segments) & $1377 \pm 72$ & $840 \pm 64$ \\
healthy controls (120 segments) & $1285 \pm 49$ & $908 \pm 94$ \\
\hline
\end{tabular}

'Ehime University Graduate School of Medicine, Toon city, Japan

Full list of author information is available at the end of the article 


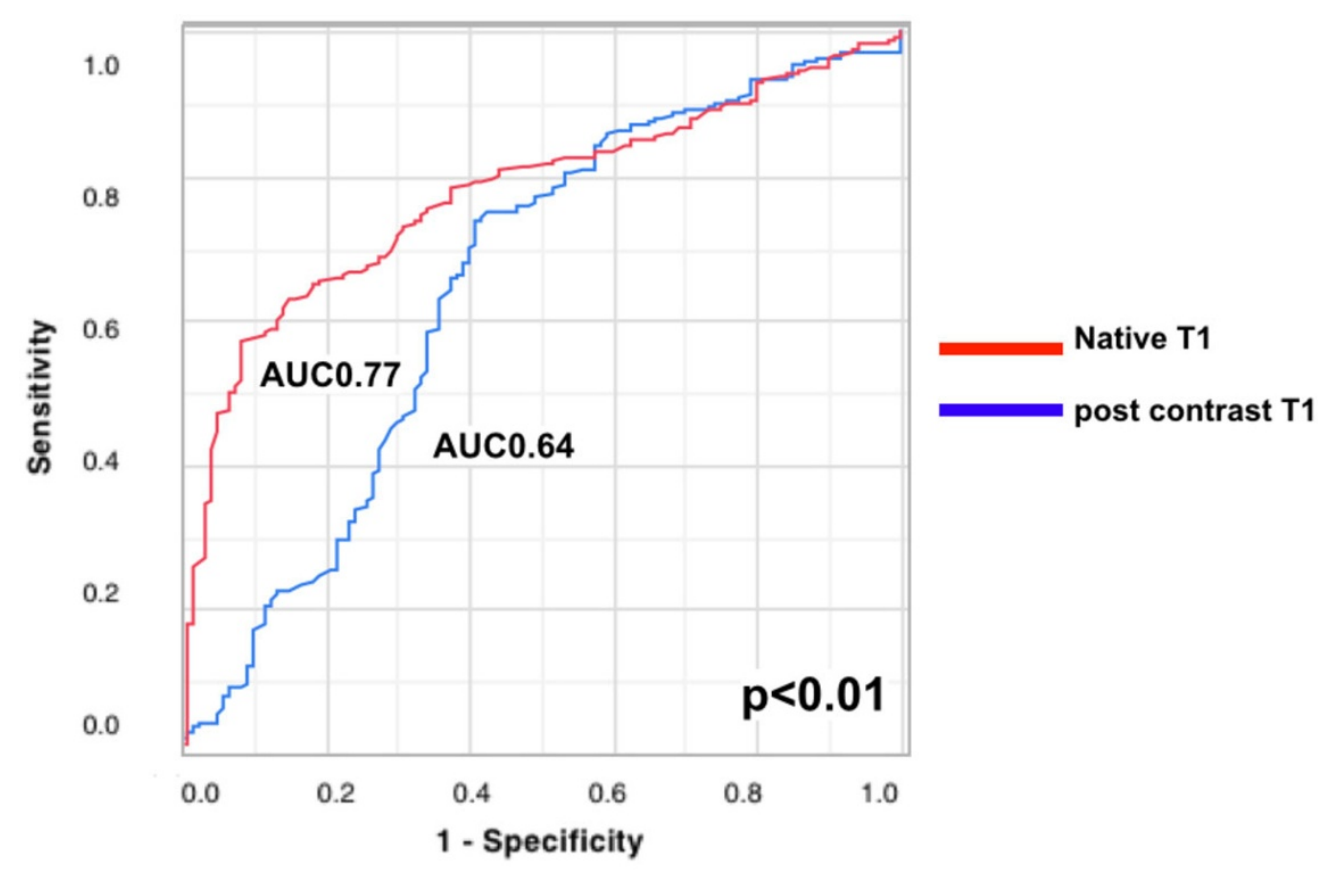

HCM : 240 segments healthy control : 120 segments

Figure 1 ROC curves for T1 values in differentiation between healthy and abnormal segments (HCM). Native T1 values using a SASHA provide better distinction between healthy and abnormal segments (HCM) than post-contrast $\mathrm{T} 1$ values.

\section{Conclusions}

Native T1 values using a SASHA provide better distinction between healthy controls and HCM. Native T1 values using a SASHA have a potential to detect myocardial fibrosis in HCM without using gadolinium contrast agents.

\section{Authors' details}

'Ehime University Graduate School of Medicine, Toon city, Japan. ${ }^{2}$ Saiseikai

Matsuyama Hospital, Matsuyama, Japan.

Published: 27 January 2016

doi:10.1186/1532-429X-18-S1-P109

Cite this article as: Ogawa et al:: T1 mapping using a saturation recovery single-shot acquisition at 3 Tesla MRI in differentiation of normal myocardium from hypertrophic cardiomyopathy. Journal of Cardiovascular Magnetic Resonance 2016 18(Suppl 1):P109.
Submit your next manuscript to BioMed Central and take full advantage of:

- Convenient online submission

- Thorough peer review

- No space constraints or color figure charges

- Immediate publication on acceptance

- Inclusion in PubMed, CAS, Scopus and Google Scholar

- Research which is freely available for redistribution 